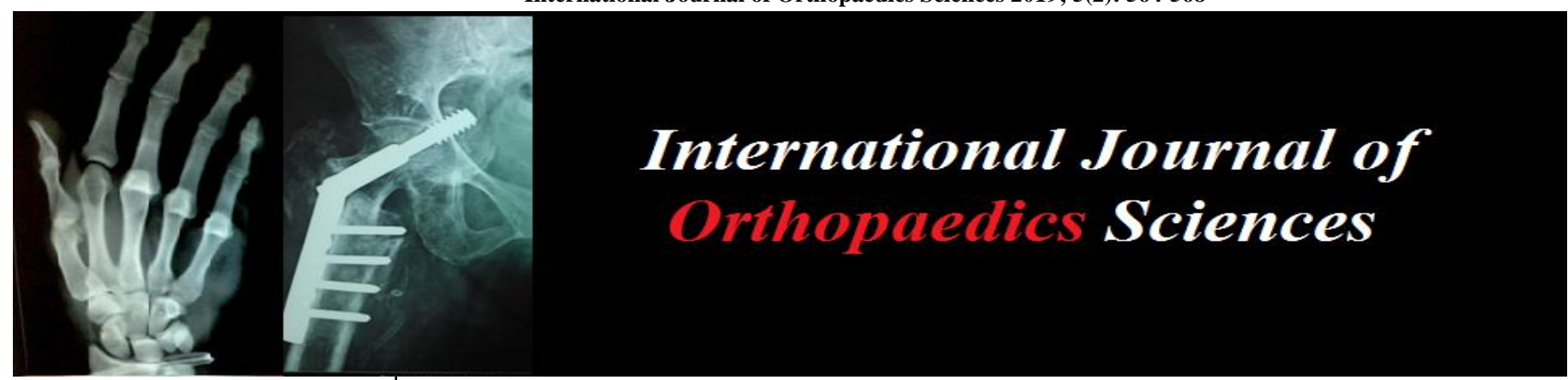

ISSN: $2395-1958$

IJOS 2019; 5(2): 564-568

(C) 2019 IJOS

www.orthopaper.com

Received: 11-02-2019

Accepted: 15-03-2019

Naik Sandeep

Asst. Professor Dept of Orthopaedics Shri. B.M. Patil

Medical College, Hospital \&

Research Centre BLDE (DU)

Vijayapura, Karnataka, India

Chamkeri Puneet

Asst. Professor Dept of Orthopaedics Shri. B.M. Patil

Medical College, Hospital \&

Research Centre BLDE (DU)

Vijayapura, Karnataka, India

Nandi Santosh

Asst. Professor Dept of

Orthopaedics Shri. B.M. Patil

Medical College, Hospital \&

Research Centre BLDE (DU)

Vijayapura, Karnataka, India

Correspondence

Naik Sandeep

Asst. Professor Dept of

Orthopaedics Shri. B.M. Patil

Medical College, Hospital \&

Research Centre BLDE (DU)

Vijayapura, Karnataka, India

\section{Clinical outcomes and complications with minimally invasive plate osteosynthesis in closed extraarticular distal tibia fractures: A prospective study}

\section{Naik Sandeep, Chamkeri Puneet and Nandi Santosh}

DOI: https://doi.org/10.22271/ortho.2019.v5.i2h.55

\section{Abstract}

Extra articular distal tibia fractures are not common injuries and are usually associated with soft tissue component. Minimally Invasive Percutaneous Plate Osteosynthesis (MIPPO) preserves the vascularity of the bone thereby achieving predictable healing. A total of 25 cases were included in the study who underwent MIPPO as treatment for the injuries using locking compression plate screws devices of fixation. Majority (92\%) of the fractures united within 16-24 weeks. Infection and impingement were the two most recognised complications in our study. Most of our cases $(90 \%)$ had excellent to good outcome. MIPPO proves to be a reliable method of fixation for these injuries and is met with good final outcome.

Keywords: Distal tibia fractures, MIPPO, locking compression plates

\section{Introduction}

Fractures of extra-articular distal tibia are among the most challenging injuries in human body. The common concern among these fractures is associated soft tissue injury component and if not treated properly may result in serious complications and disability ${ }^{[1]}$. Most often these are caused by high energy motor vehicle accidents ${ }^{[2]}$. The incidence of extra-articular distal tibia fractures in most series is $0.7 \%$ and it constitutes to about $10-13 \%$ of all tibial fractures ${ }^{[3]}$

Moreover since distal tibia is devoid of any muscular attachments even if fracture is closed there is significant soft tissue injury which needs attention while planning for treatment and proximity of fracture to plafond makes it difficult to achieve stable fixation in distal fragment with nailing and also chances of malalignment are more with nailing.

Open reduction and Plate fixation is effective in stabilizing distal tibia fractures but it is met with extensive soft tissue dissection and increased chances of infection ${ }^{[4,5]}$. Percutaneous plating techniques use indirect reduction methods and allow stabilization of distal tibia fractures while preserving vascularity of the soft-tissue envelope. The technique is demanding and requires learning curve for less chances of infection and loss of reduction ${ }^{[6,7,8]}$.

The available literature in this arena is limited and there is controversy on ideal treatment for these fractures and there are differences in clinical outcomes.

The present study was hence undertaken to study the surgical management of closed extra articular distal tibia fractures using MIPPO, the functional outcome and the various complications associated with it.

\section{Materials and Methods}

In this study, 25 skeletally mature patients with closed extra articular fractures were included and all cases presented in immediate period of trauma.

Muller et al., defined distal tibial metaphysis by constructing a square, with sides of length defined by widest portion of tibial plafond. Fractures within $5 \mathrm{~cm}$ from the tibial plafond are considered as distal tibia fractures and those not extending in to the plafond as extra articular [9] (Fig 1). 


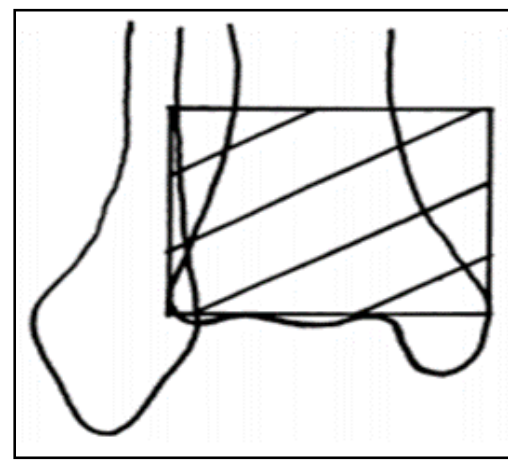

Fig 1: The plafond as extra articular

Informed consent for inclusion into the study and further treatment was taken from all the patients and ethical clearance was obtained by Institutional Ethical Committee of BLDE Shri B M Patil Medical College Hospital and Research Centre. The period of study was for 2 years from May 2015 to May 2017.

We excluded pathological fractures, open fractures and patients who were unfit for surgery. After stabilising the patient, the limb was immobilised in plaster slab and all necessary investigations for the operative procedure were ordered. Keeping in mind the precarious nature of skin in the distal tibia and its influence on further treatment the skin condition was regularly monitored and strict limb elevation was advised. Standard anteroposterior and lateral view radiographs were taken for the affected limb for planning the surgery and to decide dimensions of the implants needed.

Surgery was performed in our operative room specialised for tertiary trauma care under regional anaesthesia with a tourniquet in the supine position on a radiolucent table. All cases were operated with anatomical locking compression plate on the antero-medial surface of tibia achieving at least 6 cortices fixation on either side of the fracture. (Fig 2)

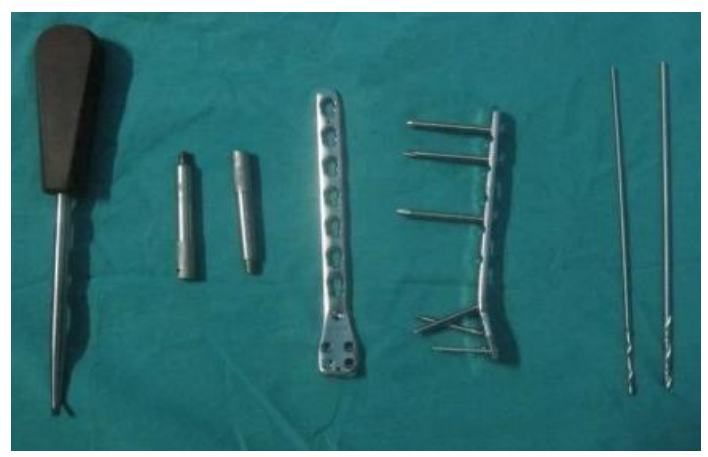

Fig 2: 6 cortices fixation on either side of the fracture

Certain steps were followed to achieve proper alignment and stability of the fixation and we followed them as a protocol in all cases:

1) Usage of wooden bolster which is radiolucent and using it as traction device for alignment and reduction.

2) Keeping a folded towel below the fracture site to prevent posterior sag of distal fragment

3) Usage of $\mathrm{k}$ wires for temporary fixation and maintaining alignment.

4) Reshaping of plate with plate bender to align the plate properly on medial surface of tibia.

5) At no cost we tried to open the fracture site for reduction purpose or tried to put a lag screw at fracture site there by disturbing the periosteal sleeve and hematoma.
6) Achieving at least 6 cortex fixation through plate on either side of fracture.

7) If needed fibular fixation to achieve stability.

8) Use of image intensifier to assist and confirm the reduction and implant position.

A 2-3 cm vertical incision was made directly over the medial malleoli for tunnelling the plate. A drill sleeve attached to plate was used to assist in tunnelling. If needed, plate was reshaped to sit properly on the surface. Plate was held to bone with $\mathrm{k}$ wires using $1 \mathrm{~mm} \mathrm{k}$ wires. Reduction was confirmed and first non-locking cortical screw was inserted in the proximal fragment of the tibia. Once the plate was snugly fit to surface, rest of the screws were distally inserted via vertical incision and proximally through small stab incisions. Special care was taken to make sure distal fragment was not angulated posteriorly thereby preventing recurvatum deformity at the ankle.

Incision was closed in layers and limb was immobilised in plaster slab. Standard antibiotic protocol of administration of IV antibiotics was practised. Sutures were removed at end of second week. We followed strict non-weight bearing protocol for a period of 6 weeks.

\section{Results}

In our study, 25 cases of closed extra articular distal tibia fractures were treated. All cases were presented in the immediate period of trauma, 12 patients were male and 13 female. The mean age of patients was 20.3 years. Out of 25 fractures, 20 were caused by road traffic accident, 4 from fall and 1 had history of assault. 12 patients had fracture on right side and 13 on left side. (Table 1).

Table 1: Characteristics of patients included in the study

\begin{tabular}{|c|c|c|}
\hline Characteristics & Number & Percentage \\
\hline \multicolumn{3}{|c|}{ Sex } \\
\hline Male & 12 & 48.0 \\
\hline Female Cause of fracture \\
\hline \multicolumn{3}{|c|}{ C } \\
\hline Road Traffic Accidents & 20 & 80.0 \\
\hline Fall & 1 & 16.0 \\
\hline Assault & 13.0 \\
\hline Right & 12 & 48.0 \\
\hline Left & 13 & 52.0 \\
\hline
\end{tabular}

All fractures were classified according to AO/OTA classification of which 3 fractures were 43A1, 10 fractures were $43 \mathrm{~A} 2$ and 12 fractures were 43A3. All fractures were closed. the soft tissue injury was graded according to Tscherene classification of which 5 fractures were type 0,9 were type 1 and another 11 were of type 2 injury. (Table 2)

Table 2: Classification of fractures

\begin{tabular}{|c|c|c|}
\hline Classification & Number & Percentage \\
\hline \multicolumn{3}{|c|}{ AO/OTA Classification } \\
\hline $43 \mathrm{~A} 1$ & 3 & 12.0 \\
\hline $43 \mathrm{~A} 2$ & 10 & 40.0 \\
\hline $43 \mathrm{~A} 3$ & 12 & 48.0 \\
\hline \multicolumn{3}{|c|}{ Tscherene Classification } \\
\hline Type 0 & 5 & 20.0 \\
\hline Type 1 & 9 & 36.0 \\
\hline Type 2 & 11 & 44.0 \\
\hline
\end{tabular}

Only one patient had associated injuries in form of posterior 
dislocation of contralateral hip, which was reduced and an open metatarsal fracture of ipsilateral foot, treated with debridement, $\mathrm{K}$ wire fixation and closed reduction of hip.

All patients were operated within 1 week of admission, except one who was operated 10 days after injury as he had associated injury detailed above.

All cases were operated using distal tibia anatomical locking plate and screws. 15 distal tibia fractures had associated fibula fracture, of which 7 were treated conservatively without fixation, 8 underwent open reduction and fixation with DCP and screws. The decision to fix the fibula was left to surgeon keeping in mind the intraoperative reduction and alignment of tibia fracture.

Patient was discharged once soft tissue has healed. Strict nonweight bearing protocol was adopted for 6 weeks.

Patients were followed up at 6 weekly intervals for evaluation of clinic-radiological signs for union. 23 out of 25 cases had signs of progress towards union and were allowed to bear weight partially. Further follow up was at 4 weekly intervals to decide on full weight bearing and to assess progression to union.

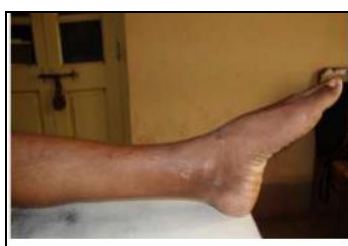

Good range of plantar flexion at end of 1 year

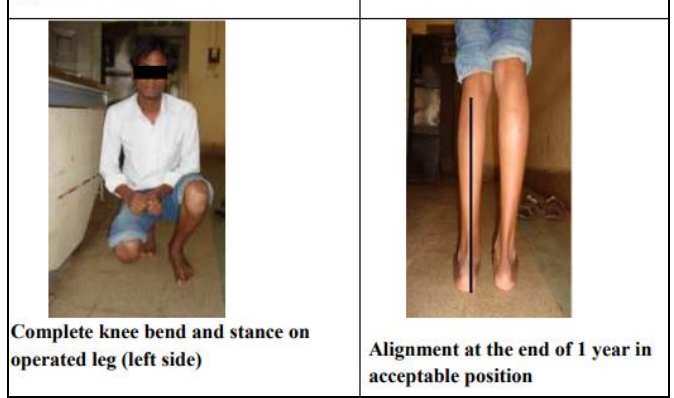

Case 1: Clinical Photograph

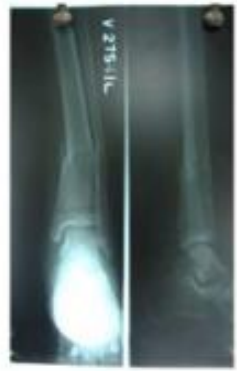

Preoperative AP and Lateral view-metaphyseal comminution present

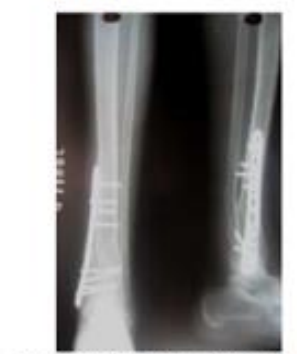

Fracture united at around 20 weeks in acceptable alignment

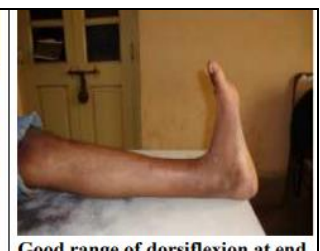

Good range of dorsiflexion at end of 1 year

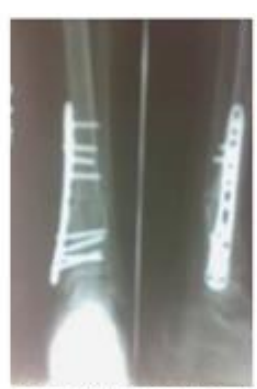

Immediate post operativ period in accepatble alignment

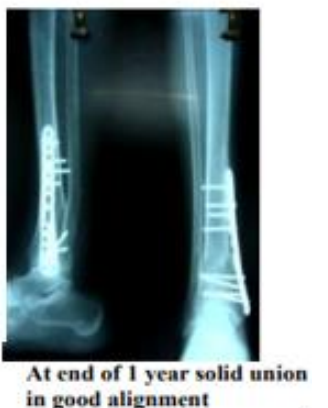

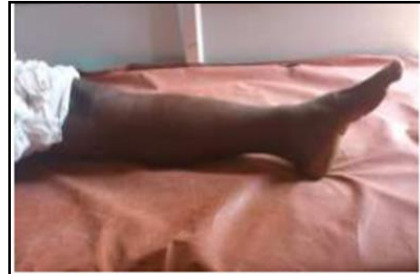

Good range of plantar flexion at end of 1 year

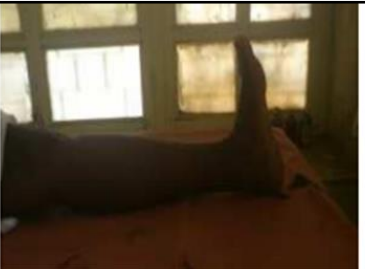

Good range of dorsiflexion at the end of 1 year

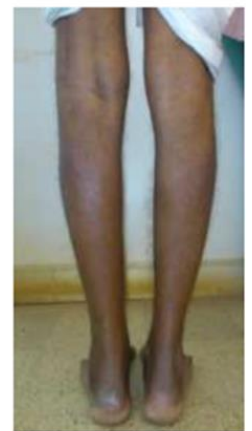

Good alignment at the end of one year (left leg operated)

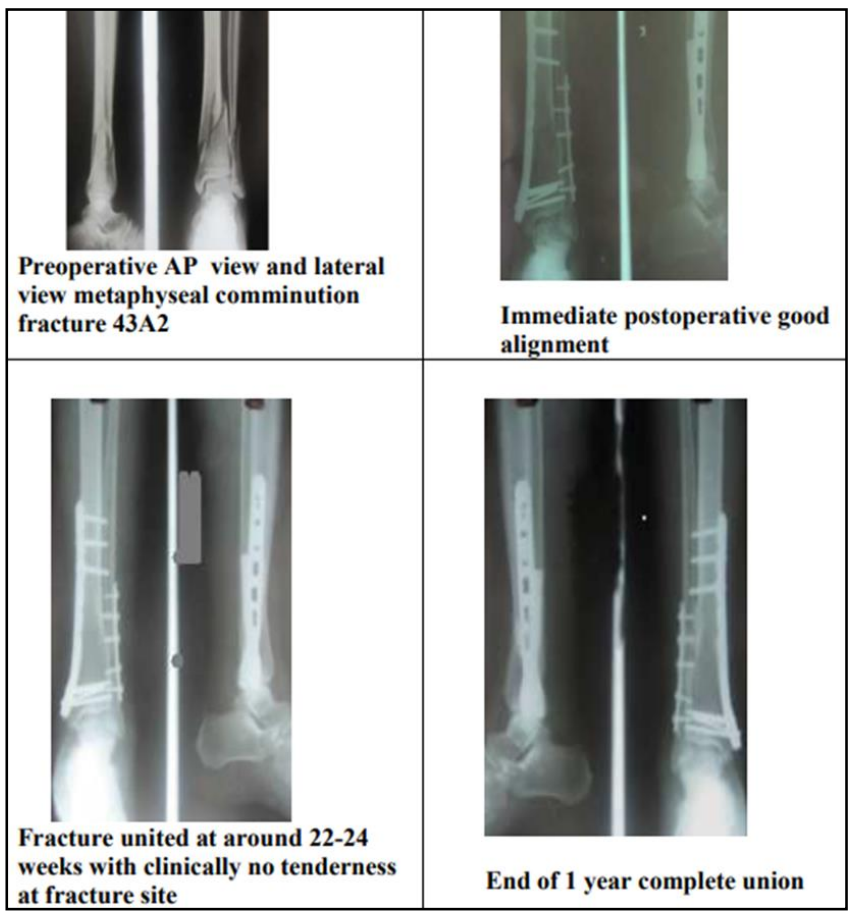

Case 2: Clinical Photograph

All fractures united in this study and most of them (92.0\%) united between 16-24 weeks. The mean union time was 20 weeks. 2 fractures showed delayed union with no signs of union by end of 12 weeks. Both these fractures which had slow union were not having clinical symptoms. They were treated with bone marrow injection to the fracture site from iliac crest under aseptic precautions. Both progressed to union at 24 weeks.

Superficial infection at distal screw site was seen in 1 case on $8^{\text {th }}$ day of post-operative period. This was managed with continuation of IV antibiotics and local debridement which eventually healed. Deep Infection occurred in one case which presented at $8^{\text {th }}$ month of follow up with signs of cellulitis, skin breakdown and sinus at implant site with implant exposed. Since fracture was united, implant removal was done and case was managed with culture sensitive antibiotics. 2 cases had impingement and implant irritation at proximal site 
for which counselling done. 1 case had fibula fixation related complication in the form of wound breakdown and distal screw expose, which was treated with screw removal under local anaesthesia and antibiotics. (Table 3) The minimum period of follow up in our study was 24 months.

A clinical assessment of ankle function according to the criteria of Merchant and Dietz was determined for each ankle at the end of 1-year follow-up. It is a 100-point scale allotting 40 points for function, 40 for pain, 10 for gait and 10 points for range of motion at the ankle. A score of 85-100 represents an excellent result, 70-84 good result, 60-69 fair result and less than 60 poor results. 6 had excellent outcome, 16 had good and 3 had fair.

Table 3: Outcome and Complications of patients included in the study

\begin{tabular}{|c|c|c|}
\hline Outcome/Complications & Number (Percentage) & Treatment Opted \\
\hline Complete Union & $23(92.0)$ & - \\
\hline Delayed Union & $2(8.0)$ & Bone marrow injection \\
\hline Superficial infection at distal screw site & $1(4.0)$ & IV antibiotics and local debridement \\
\hline Deep Infection at implant site with implant exposed & $1(4.0)$ & Implant removal as fracture was united \\
\hline Impingement and implant irritation at proximal site & $2(8.0)$ & Counselling \\
\hline Fibula fixation complication -wound breakdown and distal scr & $1(4.0)$ & Screw removal \\
\hline
\end{tabular}

\section{Discussion}

The optimal management of extra articular distal tibia fractures continues to be a controversial topic because

1. They are caused by high energy trauma.

2. Proximity to plafond.

3. Soft tissue injury around the fracture site.

Careful preoperative planning is needed in consideration with above mentioned factors in selecting the correct implant for given fracture pattern and associated soft tissue injury and minimizing the postoperative malalignment and soft tissue complication ${ }^{[10]}$

Understanding of the mechanical background for choosing the proper implant length and the type and number of screws is essential to obtain a sound fixation with a high plate span ratio and a low plate screw density. A high plate span ratio decreases the load onto the plate. A high working length of the plate reduces the screw loading, thus fewer screws need to be inserted and the plate screw density can be kept low ${ }^{[10]}$.

In our study most common cause for these fractures was road traffic accident (R.T.A). Most of the standard studies report vehicular accidents as the common mode of injury followed by fall. Standard studies have classified these fractures according to AO/OTA classification and soft tissue injury using Tscherene classification. In our study we have used AO/OTA classification system for fracture description and grading of soft tissue injury according to Tscherene classification thereby eliminating intra and inter observer variability ${ }^{[11]}$.

Most of the cases in our study were operated between 3-7 days of injury. This window period was given for abrasion over the skin to heal and swelling of the ankle to reduce. These steps were taken to reduce the risk of soft tissue complications associated with these fractures. The injurysurgery interval time was 3-7 days in study by Ashwin et al. ${ }^{[10]}, 6$ days in study by Ronga $\mathrm{M}$ et al. ${ }^{[12]}$ and within 7 days by Siddharth et al. ${ }^{[13]}$ which was similar to our study.

In our study, we allowed weight bearing only after signs of union in form of bridging callus was present on at least 3 cortices on radiograph and absence of tenderness at fracture at the end of 6 weeks. 23 out of 25 cases met such criteria and were allowed partial weight bearing.

The mean time of union in our study was 20 weeks. We achieved union in all 25 cases treated with MIPPO within 24 weeks. Mean union time for the fractures treated with MIPPO was 15.2 weeks (range, 10-24 weeks) by Oh W, et al. ${ }^{[14]}$ and by 24 weeks in study by Ronga et al. ${ }^{[12]}$ Borg $\mathrm{T}$ et al. ${ }^{[15]}$ and 22 weeks by Somashekhar et al. ${ }^{[16]}$ as seen in our study where as the mean time for union in Collinge C,et al. ${ }^{[17]}$ study was 35 weeks but all fractures which were closed healed within 25 weeks.

Two cases in our study had problems with infection one had superficial and another had deep infection presenting as discharging sinus. Comparable to our work, Lau T, et al. ${ }^{\left[{ }^{8]}\right.}$ in their study of 48 cases had 4 such deep infection cases (10\%) occurring between 2-12 months after fixation and they believe infection does not have effect on bone healing. So it can be concluded that if deep infection is present and fracture is consolidated sufficiently, the implant can be removed.

Deep infection was seen in one patient $(4.76 \%)$, superficial infection in three patients $(14.28 \%)$ in plating group by Davalgapu et al. ${ }^{[18]}$. and study by Ehlinger M, et al. ${ }^{[19]}$ had 2 deep infections (5\%) requiring lavage and change of osteosynthesis and Guo J, et al. ${ }^{[20]}$ had reported $6 \%$ of cases had deep infection which merited intervention. Studies by Somashekhar et al. ${ }^{[16]}$ and Bahari et al. ${ }^{[21]}$ reported 2 cases of superficial infection and one case of deep infection.

None of the cases had implant failure as complication in our study. In studies conducted by $\mathrm{Oh} \mathrm{W}$, et al. ${ }^{[14]}$, Ehlinger $\mathrm{M}$, et al. ${ }^{[19]}$ and Nork S, et al. ${ }^{[22]}$ no complications related to implant failure occurred. However, study by Collinge C, et al. ${ }^{[17]}$ had $7 \%$ cases with loss of fixation. No implant related complications occurred in our study probably due to strict non weight bearing post-operative protocol.

We had 2 cases of implant impingement and irritation in 2 of 5 cases of MIPPO for which symptomatic treatment was given and patients are doing well. Oh $\mathrm{W}$, et al. ${ }^{[14]}$ in their study had $40 \%$ patients experiencing implant discomfort and opined that implant irritation and impingement is related to thickness of implant and better designs will solve this problem. Lau T, et al. ${ }^{[8]}$ in their study had 52\% patients experiencing implant impingement. Only $14 \%$ had their implants were removed for this reason and opined that decision on implant removal for this complication should be justified.

The mean ankle evaluation score at the end of one year using Iowa ankle score by Merchant and Dietz ${ }^{[23]}$ was 78 (ranging from 63-82) and this corresponds to overall good result. $85 \%$ of the cases in our study had excellent to good results. In our study none of the cases had poor outcome. Oh W, et al. ${ }^{[14]}$ had excellent to satisfactory results in all cases using Olerud score at the end of mean follow up of 20 months. Guo J, et al. ${ }^{[20]}$ in their work had mean score 81 for MIPPO using AOFAS as scoring system for ankle function which corresponds to good outcome. Collinge C, et al. ${ }^{[17]}$ in their study used both AOFAS and Olerud score to evaluate functional outcome and 
had good results in most of their cases. In study by Ehlinger M, et al. ${ }^{[19]}$, using Olerud score good results were seen in $80 \%$ of cases.

\section{Conclusion}

Minimally invasive plate osteo synthesis by locking plate proves to be a reliable method of fixation for extra articular distal tibial fractures. This procedure preserves most of the osseous vascularity, fracture hematoma which provides biological repair so that there is lesser incidence of delayed union and non-union. This technique can be used in fractures where locked nailing cannot be done like distal tibial fractures with small distal metaphyseal fragments and comminuted fractures. Management of these fractures are not without complications such as infection and delayed union; hence these complications should be looked for at the earliest and treated accordingly. If principles of treatment are correctly followed, most cases will progress to union and will have good final outcome with acceptable and predictable complications.

\section{References}

1. Stannard J, Schmidt A, Kregor P. Surgical treatment of orthopaedic trauma, $1^{\text {st }}$ edition. 2007, 767-97.

2. Schatzker J, Tile M. The rationale of operative fracture care, $3^{\text {rd }}$ edition, 475-76.

3. Court-Brown Caesar B. Epidemiology of adult fractures: A review. Injury. 2006; 37:691-697.

4. Borrelli J, Prickett W, Song E, Becker D, Ricci W. Extraosseous blood supply of the tibia and the effects of different plating techniques: a human cadaveric study. J Orthop Trauma. 2002; 16(10):691-5.

5. Bedi A, Toan T, Karunakar M. Surgical Treatment of Nonarticular Distal Tibia Fractures. J Am Acad Orthop Surg. 2006; 14(7):406-16.

6. Extraarticular distal-tibial fractures: Plating or intramedullary nailing?. Orthop trauma dir. 2009; 06:1928.

7. Ghera S, Santori F, Calderaro M, Giorgini T. Minimally Invasive Plate Osteosynthesis in Distal Tibial Fractures: Pitfalls and Surgical Guidelines. Trauma Orthop 2004; 27(9):903.

8. Lau T, Leung F, Chan F, Chow S. Wound complication of minimally invasive plate osteosynthesis in distal tibia fractures. Int Orthop 2008; 32(5):697-703.

9. Müller M, Nazarian S, Koch P, et al. The Comprehensive Classification of Fractures of Long Bones. SpringerVerlag, 1990.

10. Mudgal A, Daolagupu A, Agarwal V, Sinha A. Management of Fractures of the extra-articular distal tibia by minimally invasive plate osteosynthesis-A prospective series of 21 patients. Int J Med Research \&Health Sciences. 2016; 5(6):276-282.

11. Chapman MW. Fractures of the tibial and fibular shafts. In: Chapman's orthopaedic surgery.3rd edition. Philadelphia: Lippincott Williams and Wilkins. 2001; 1:755-810.

12. Ronga M, Longo U, Maffulli N. Minimally Invasive Locked Plating of Distal Tibia Fractures is safe and Effective. Clin Orthop Relat Res. 2010; 468(4):975-982.

13. Paluvadi S, Lal H, Mittal D, Vidyarthi K. Management of fractures of the distal third tibia by minimally invasive plate osteosynthesis $-\mathrm{A}$ prospective series of 50 patients. J. Clin Ortop Trauma. 2014; 5(3):129-136.

14. Oh W, Kyung S, Park H et al. Distal tibia metaphyseal fractures treated by percutaneous plate osteosynthesis. Clin Orthop. 2003; 408:286-291.

15. Borg T, Larsson S, Lindsjö U Percutaneous plating of distal tibial fractures. Preliminary results in 21 patients. Injury. 2004; 35(6):608-14.

16. Somashekar, Girish S, Sridharamurthy, Prajwal KN. A study of functional outcome of distal tibial extraarticular fracture fixed with locking compression plate using MIPPO technique. Nat J Clin Orthop. 2017; 1(3):13-18.

17. Collinge C, Kuper M, Larson K, Protzman R. Minimally Invasive Plating of High-Energy Metaphyseal Distal Tibia Fractures. J Orthop Trauma. 21(6):355-360.

18. Davalagapu AK, Mudgal A, Agarwala V, Dutta KK. A comparative study of intra medullary inter locking nailing and minimally invasive plate osteosynthesis in extra articular distal tibia fractures. Indian J Orthop. 2017; 51:292-8.

19. Ehlinger M, Adam P, Gabrion A, Jeunet L, Dujardin F, Asencio G Sofcot. Distal quarter leg fractures fixation: The intramedullary nailing alone option. Orthop Traumatol Surg Res. 2010; 96(6):674-82.

20. Guo J, Tang N, Yang H, Tang T. A prospective, randomised trial comparing closed intramedullary nailing with percutaneous plating in the treatment of distal metaphyseal fractures of the tibia. J Bone Joint Surg Brit. 2010; 92(7):984-988.

21. Bahari et al. Minimally invasive percutaneous plate fixation of distal tibia fractures. Acta Orthop. Belg. 2007; 73:635-640.

22. Nork S, Schwartz A, Agel J, Holt S, Schrick J, Winquist R. Intramedullary nailing of distal metaphyseal tibial fractures. J Bone Joint Surg Am. 2005; 87(6):1213-21.

23. Merchant T, Dietz F. Long term follow up after fractures of the tibial and fibular shafts. J Bone Joint Surg Am. 1989; 71:599-606. 